\title{
Estrategias para la prevención y control de las enfermedades parasitarias de la tilapia
}

\author{
Strategies for prevention and control of parasite diseases in cultured tilapia
}

Emma Josefina Fajer-Ávila1* ${ }^{1 *}$, Rosa María Medina-Guerrero ${ }^{1}$, Francisco Neptalí Morales-Serna ${ }^{1}$

\section{RESUMEN}

Con base en una revisión de la literatura, el presente trabajo aborda de manera general el impacto de los parásitos en el cultivo de tilapia. Se describen algunas estrategias, incluyendo la aplicación de tratamientos naturales como una alternativa a los agentes químicos o fármacos, que deberían considerarse para la prevención de eventos epidémicos. Sin duda, los desarrollos biotecnológicos que garanticen la prevención y control de enfermedades, sin poner en riesgo la salud humana o del ecosistema, deberían prevalecer en la acuicultura de la tilapia.

PALABRAS CLAVE

piscicultura, agua dulce, peces, Cichlidae, Oreochromis, salud

\section{ABSTRACT}

The aim of the present paper is to provide an overview of the impact of parasites on farmed tilapia. We also include generalities regarding strategies for control or prevention of parasitic diseases such as the use of plant extracts as an alternative to common drugs, which could be considered for prevention of epidemic episodes. Safe, biotechnological products that guarantee prevention or control of fish diseases should be increased to support tilapia culture in Mexico.

KEYWORDS

aquaculture, freshwater, fish, Cichlidae, Oreochromis, health

\footnotetext{
${ }^{1}$ Centro de Investigación en Alimentación y Desarrollo, A. C. Unidad Mazatlán en Acuicultura y Manejo Ambiental.

*Autor para correspondencia. Centro de Investigación en Alimentación y Desarrollo, A. C. Unidad Mazatlán en Acuicultura y Manejo Ambiental. Av. Sábalo Cerritos s/n. 82100 Mazatlán, Sinaloa, México.

Correo electrónico: efajer@ciad.mx
} 


\section{INTRODUCCIÓN}

El número de granjas de tilapia, Oreochromis spp. (Perciformes: Cichlidae), y sus volúmenes de producción por acuicultura en México han mostrado un aumento sostenido a partir de 2004. Según datos de la Comisión Nacional de Acuacultura y Pesca (Conapesca, 2015), en 2014, la producción alcanzó 121,529 toneladas. Los principales estados productores de tilapia en México son Jalisco, Chiapas, Michoacán y Veracruz. En comparación con otros peces, la tilapia es más tolerante a condiciones ambientales desfavorables y a otros factores estresantes, lo cual ha facilitado su cultivo en densidades cada vez mayores y en sistemas de recirculación. Pocos agentes infecciosos son específicos para la tilapia; sin embargo, en ciertas condiciones, algunos patógenos la afectan de manera más severa que a otras especies de peces. Las temperaturas bajas, el manejo inadecuado, la calidad pobre del agua (oxígeno bajo, alto contenido de amonio, nitritos o $\mathrm{CO}_{2}$, niveles de $\mathrm{pH}$ adversos) y la alimentación inadecuada reducen la inmunidad y comprometen la resistencia de la tilapia a las enfermedades, lo que da lugar a una mayor incidencia de infecciones (El-Sayed 2006). El objetivo del presente estudio es resumir las principales enfermedades parasitarias que afectan a la tilapia en México y las estrategias para su prevención y control.

\section{Patógenos frecuentes}

La tilapia no es muy susceptible a los procesos virales. Entre los que afectan a las tilapias silvestres está la linfocistis, que ocasiona papilomas en piel y aletas (Paperna, 1973), pero su prevalencia es baja en México. También se conoce la encefalitis viral en larvas de tilapia Oreochromis aureus (TLEV) en Israel, causante de altas mortalidades y transmisible a otras especies de tilapia (Shlapobersky et al., 2010). Recientemente, se registró el Tilapia Lake Virus (TiLV) (Eyngor et al., 2014), que provoca mortalidades significativas (80\%) en alevines silvestres y cultivados en Israel; sin embargo, hasta la fecha, éste no se ha registrado en México. Por otro lado, en el cultivo de tilapia es frecuente encontrar una amplia variedad de organismos oportunistas tales como ciliados y monogeneos parásitos, cuyo efecto sinérgico con las bacterias ubicuitarias no debe ser subestimado.

Los tricodínidos son los ciliados predominantes en las tilapias; se incrementan dramáticamente en invierno y pueden afectar tanto la piel como las branquias. La abundancia de estos ciliados se asocia con una pobre calidad del agua y, aunque no son particularmente patógenos, sus estructuras quitinosas pueden causar irritación e inflamación de la piel, y con ello propiciar la entrada de bacterias causantes de enfermedades (Johnson, 2000; Xu et al., 2015). En sistemas de recirculación intensivos, los niveles altos de infestación por tricodínidos dañan la epidermis de la tilapia facilitando el incremento de infecciones por Streptococcus.

Entre los monogeneos, existen diferentes especies de Gyrodactylus que parasitan frecuentemente las aletas, superficie de la piel, ojos y branquias de la tilapia y pueden causar gyrodactylosis crónica bajo condiciones de cultivo. En México, se han registrado las especies G. cichlidarum y G. yacatli en Oreochromis niloticus (García-Vásquez et al., 2007, 2011). Estos parásitos tienen la habilidad de incrementar sus poblaciones en un periodo de tiempo corto debido a su alta tasa de natalidad y a la transmisión directa entre las tilapias (Rawson y Rogers, 1973). Procesos epidémicos por gyrodactylosis se han desarrollado en larvas de tilapia cultivadas en varios estados de México.

Las lesiones que ocasionan los parásitos son portales potenciales para la entrada de bacterias patógenas. Estas lesiones pueden permitir la invasión bacteriana, lo que provoca que los peces con G. cichlidarum sean más susceptibles a la bacteria Streptococcus iniae. Por microscopía electrónica se han observado colonias bacterianas sobre la superficie de Gyrodactylus, lo que sugiere que estos macroparásitos pueden actuar como vectores de bacterias (Cusack y Cone, 1985). Además, estudios experimentales han demostrado el efecto sinérgico de los monogeneos gyrodactílidos en el desarrollo de enfermedades bacterianas como la estreptococosis (Xu et al., 2007). En un sistema de cultivo en jaulas flotantes en Sinaloa, se detectaron mortalidades de tilapia de hasta $20 \%$ por jaula, asociadas a la presencia abundante de Gyrodactylus y Streptococcus. Las tilapias mostraron nado errático, lesiones hemorrágicas en el cuerpo, exoftalmia y opacidad de la córnea (E. J. Fajer-Ávila, observación personal).

Ichthyophthirius multifiliis y Streptococcus iniae son patógenos importantes de la tilapia nilótica en cultivo, en especial cuando disminuye la temperatura. En comparación con otros peces de aguas cálidas, la tilapia es más resistente al "Ich"; sin embargo, cuando las temperaturas son óptimas $\left(20-25^{\circ} \mathrm{C}\right)$ y las densidades de siembra son altas, pueden ocurrir infecciones severas y pérdidas importantes causadas por este patógeno. Streptococcus iniae es responsable 
de mortalidades de $75 \%$ en sistemas de cultivo cerrados (Agnew y Barnes, 2007). Al igual que en el caso de Gyrodactylus, las coinfecciones experimentales Ich-Streptococcus pueden incrementar la mortalidad de la tilapia de 38 a $88 \%$ (Xu et al., 2009).

Investigaciones recientes han demostrado que aislados bacterianos de procesos septicémicos en O. niloticus cultivada en jaulas flotantes e inoculada experimentalmente en O. mossambicus ocasionaron $100 \%$ de mortalidad. Las bacterias aisladas fueron identificadas como Aeromonas dhakensis y Pseudomonas mosselli (Soto-Rodríguez et al., 2013), las cuales han sido catalogadas como un riesgo para el cultivo de tilapia. En tilapia de cultivo, la mala calidad del agua se ha relacionado con la aparición frecuente de la columnariasis causada por Flavobacterium columnaris, la cual ocasiona áreas necróticas sobre la superficie corporal, branquias y aletas. Infecciones por Staphylococus también se han encontrado en $O$. niloticus causando hemorragia interna y úlceras en mandíbula (S. Soto-Rodríguez, observación personal).

\section{Medidas De BIOSEguridad}

La introducción y la dispersión de parásitos y bacterias en los sistemas de cultivo de tilapia se evitan mediante el cumplimiento estricto de las medidas de bioseguridad (García y Calvario 2007); es decir, mediante la aplicación del conjunto de prácticas de manejo enfocadas en la protección (reducir la introducción y transmisión de microorganismos) y la prevención (mejorar las condiciones de vida de los peces e incrementar la resistencia del organismo al desarrollo de enfermedades).

El agua es la vía principal de entrada de huevos, estadios infectivos y vectores de patógenos, tales como caracoles y peces silvestres. Por esto, cuando su procedencia no es un manantial sino una presa o río, la colocación de filtros en la fuente de entrada es imperativa. El suministro de alimento balanceado de acuerdo con la edad y los requerimientos de la especie garantiza un buen estado de salud; no obstante, éste debe estar bien almacenado para evitar el desarrollo de hongos productores de aflatoxinas que pueden afectar el crecimiento y desencadenar mortalidades en las tilapias. Las larvas también deben estar libres de parásitos debido a que su sistema de defensa no está completamente desarrollado y son susceptibles a enfermedades. En este sentido, la técnica de incubación de huevos en las jarras Mc Donald para la obtención de larvas de tilapia evita que los progenitores portadores de tricodínidos infecten a su descendencia.
Los procedimientos de bioseguridad deben ser siempre considerados en combinación con un programa efectivo de higiene, formado básicamente por los procesos de limpieza y desinfección adecuada a las condiciones locales (García y Calvario, 2007).

Para evitar la introducción y diseminación de enfermedades, los Comités de Sanidad Acuícola de los diferentes estados del país promueven programas de vigilancia y monitoreo de patógenos y el movimiento responsable de organismos. La detección temprana de patógenos permite la aplicación de las medidas terapéuticas para su control. La expedición de certificados de salud con anterioridad a la exportación o traslado de los peces entre granjas es un requisito de control que no se debe obviar. De igual forma, para el caso de los peces importados, es importante exigir un certificado de salud de alguna institución de sanidad reconocida para evitar la introducción de patógenos exóticos. La cuarentena es un aspecto relevante que no se ha de soslayar para los peces importados. En la cuarentena se deben garantizar las revisiones al inicio y al final de un periodo establecido -de por lo menos 21 días-, lo cual permitiría detectar la introducción de patógenos. Los esfuerzos combinados no reducen solamente el daño directo de los parásitos sino también minimizan las mortalidades debido a infecciones bacterianas secundarias (Xu et al., 2009).

\section{Prevención y CONTROL DE LAS ENFERMEdAdes}

Para tratar enfermedades en la acuicultura, existe un gran interés a nivel mundial por restringir el uso de agentes químicos y fármacos, ya que estas sustancias pueden ser perjudiciales tanto para los peces como para los consumidores y el medio ambiente (Cabello, 2006); además, requieren de un tiempo de retiro, lo que restringe la comercialización del producto para consumo humano.

Los productos fitofarmacéuticos han sido reconocidos como la terapia del futuro para la prevención y control de las enfermedades de organismos acuáticos. La mayor parte de las plantas medicinales son frecuentes en los países tropicales y subtropicales, y su efecto terapéutico y profiláctico en especies acuáticas ha sido validado en México, Cuba, India, Tailandia, Japón y Turquía (Prieto et al., 2005; Yin et al., 2005; Aly et al., 2008; Ardó et al., 2008; Wu et al., 2010; del Rio-Zaragoza et al., 2011). Sin embargo, las investigaciones en este campo y su empleo en la acuicultura son aún insuficientes.

En los últimos años, se ha incrementado la búsqueda de compuestos naturales extraídos de 
plantas que tengan beneficios para los peces y los hagan más resistentes a enfermedades. Algunos de estos compuestos, llamados inmunoestimulantes, son considerados promotores de la salud porque potencializan la respuesta inmune inespecífica en los organismos de manera profiláctica. Los mecanismos que componen la respuesta inmune inespecífica proveen protección al hospedero evitando la adhesión, invasión o multiplicación de patógenos mediante diferentes tipos de barreras: físicas (piel, branquias, intestino), humorales (complemento, transferrinas, antiproteasas, hemolisinas, lisozimas, interferón, proteína $\mathrm{C}$ reactiva) y celulares (leucocitos, incluyendo macrófagos y neutrófilos); además del proceso de inflamación. Otro efecto deseable en la administración de inmunoestimulantes dietarios es que provean mejor crecimiento, supervivencia y ganancia en peso; es decir, que su uso en peces se refleje en mejores indicadores de productividad.

Algunos extractos de plantas incrementan los mecanismos de defensa inespecífica (número de leucocitos, lisozima sérica, actividad fagocítica, proteína sérica, globulinas, respuesta inflamatoria) y la resistencia natural de la tilapia frente a algunos patógenos. Estos extractos se han administrado vía intraperitoneal a nivel experimental y vía oral a escala piloto (Gioacchini et al., 2008). En el cuadro 1 se resumen algunos resultados del efecto dietario de extractos de plantas en tilapia sobre los principales indicadores de respuesta inmune para diferentes periodos de inclusión.

Las algas despiertan gran interés porque constituyen una fuente natural de compuestos con actividad biológica que pueden utilizarse como ingredientes funcionales en dietas. La bioactividad de las algas o su capacidad para estimular el sistema inmune se encuentra en algunas sustancias, incluidos los extractos de polisacáridos (Costa, 2010). Estudios in vitro mostraron que el $\beta$-glucano obtenido de Laminaria hyperborean incrementó la actividad de macrófagos del salmón. Asimismo, los alginatos presentes en algas pardas e inyectados vía intraperitoneal a la trucha arcoíris aumentaron la actividad fagocítica, estallido respiratorio y expresión de citocinas en leucocitos periféricos (Peddie et al., 2002). En carpas inyectadas con carragenina -polisacárido obtenido del alga roja Chondrus ocellatus-, se observó mayor resistencia a las bacterias Edwarsiella tarda y Aeromonas hydrophilla (Fujiki et al., 1997). Diferentes especies de algas verdes del género Ulva y Enteromorpha modularon la función de los macrófagos del rodaballo (Castro et al., 2004), donde el ulvan, el ácido urónico, la xilosa, la ramnosa y otros polisacáridos ácidos sulfatados son los responsables de este efecto (Leiro et al., 2007).

Ulva clathrata (antes conocida como Enteromorpha clathrata) o Ao-nori, es una macroalga verde de la familia Ulvaceae de distribución mundial. Resultados experimentales sobre el efecto dietario del extracto de $U$. clathrata en la ración alimenticia diaria de juveniles de Oreochomis niloticus incrementó la capacidad de respuesta del sistema inmune no específico, lo que indica las ventajas de su empleo en el cultivo de esta especie (Quezada-Rodríguez, 2012).

El empleo de vacunas en peces se encuentra en una fase temprana de desarrollo enfocada principalmente en la prevención de los procesos virales y bacterianos. La empresa farmacéutica PHARMAQ, especializada en la acuicultura, se ha enfocado en el desarrollo de vacunas para mejorar la salud en tilapias. En 2014, esta empresa introdujo al mercado la primera vacuna, ALPHA JECT ${ }^{\circledR}$ micro1 TiLA, para combatir la estreptococosis en tilapia. Ésta es una vacuna inactivada monovalente oleosa, que otorga $60 \%$ de protección contra Streptococcus agalactiae, causante de altas mortalidades en tilapias cultivadas. El uso de esta vacuna ha sido aprobado y cuenta con registro en Costa Rica y Panamá.

La terapia para el control de las enfermedades de los peces es una herramienta útil para reducir las mortalidades y los bajos rendimientos. Sin embargo, antes de una terapia, se debe evaluar la tolerancia de los peces y la inocuidad del medicamento; la calidad del agua y su interacción con el compuesto, y la efectividad contra el patógeno que se va a tratar. Un aspecto importante de considerar es el costo del tratamiento antes de decidir su aplicación. La terapia se debe aplicar preferiblemente durante los inicios de la enfermedad, en larvas y alevines. Cuando los peces están listos para ser cosechados, hay que evaluar la rentabilidad de su empleo o proceder a su cosecha siempre que el patógeno sea inocuo al consumidor (Arthur et al., 2012).

En el mundo, existen diferentes tipos de regulaciones que determinan la disponibilidad de las drogas para ser usadas en especies de cultivo. En algunos países hay un mayor número de fármacos disponibles; en otros, la disponibilidad está restringida por normas estrictas orientadas hacia una mayor seguridad para el consumidor.

En Estados Unidos, el Centro de Medicina Veterinaria (CVM) de la FDA (Agencia de Administración de Drogas y Alimentos) regula la manufactura, distribución y uso de drogas en especies animales, garantizando que sean seguras, efectivas e inocuas. Entre los fármacos aprobados por la FDA, se tienen tres productos comerciales a base de $37 \%$ 
Cuadro 1. Efecto dietario de extractos de plantas en tilapia.

\begin{tabular}{|c|c|c|c|}
\hline EsPeCIE & EXTRACTO & $\begin{array}{l}\text { INCLUSIÓN EN DIETA - } \\
\text { TIEMPO DE EXPOSICIÓN }\end{array}$ & INCREMENTO EN RESPUESTA INMUNE \\
\hline O. niloticus & $\begin{array}{l}\text { Producto elaborado } \\
\text { con Astragalus radix y } \\
\text { Scutellari radix }\end{array}$ & $0.1,0.5,1 \%$ - 30 días & $\begin{array}{l}\text { Fagocitosis, lisozima y actividad de } \\
\text { estallido respiratorio. }\end{array}$ \\
\hline O. niloticus & $\begin{array}{l}\text { Producto acuoso } \\
\text { elaborado con Astragalus } \\
\text { membranaceuss y Lonicera } \\
\text { japonica }\end{array}$ & $0.1 \%$ - 30 días & $\begin{array}{l}\text { Fagocitosis, lisozima, anticuerpos y } \\
\text { resistencia a Aeromona hydrophila. }\end{array}$ \\
\hline O. mossambicus & $\begin{array}{l}\text { Extracto de } \\
\text { lechuga (Eclipta alba) }\end{array}$ & 0.1 a $1 \%$ - 21 días & $\begin{array}{l}\text { Actividad de lisozima, producción de } \\
\text { oxígeno reactivo y mieloperoxidasa, } \\
\text { resistencia a } A \text {. hydrophila. }\end{array}$ \\
\hline O. niloticus & $\begin{array}{l}\text { Extracto de Laminaria } \\
\text { japónica (Laminarían) }\end{array}$ & $0.1 \%$ - 21 días & $\begin{array}{l}\text { Transformación linfocitaria, actividad } \\
\text { fagocítica, recuento de linfocitos, } \\
\text { actividad bactericida del suero, oxido } \\
\text { nítrico y resistencia a } A \text {. hydrophila }\end{array}$ \\
\hline O. mossambicus & $\begin{array}{l}\text { Extracto de semilla en } \\
\text { cloroformo de Nyctanthes } \\
\text { arbortristis }\end{array}$ & $0.01,0.1,1 \%$ - 21 días & $\begin{array}{l}\text { Lisozima, complemento, } \\
\text { meloperoxidasa y especies de oxígeno } \\
\text { y nitrógeno reactivo. Resistencia a } A \text {. } \\
\text { hydrophila. }\end{array}$ \\
\hline O. niloticus & $\begin{array}{l}\text { Extracto de L. japonica y } \\
\text { Ganoderma lucidum }\end{array}$ & $0.5,1 \%$ - 21 días & $\begin{array}{l}\text { Fagocitosis, actividad de lisozima y } \\
\text { resistencia a } A . \text { Hydrophila. }\end{array}$ \\
\hline O. niloticus & $\begin{array}{l}\text { Extracto acuoso de } \\
\text { Andrographis } \\
\text { paniculata }\end{array}$ & $0.5,1,1.5 \%$ - 30 días & $\begin{array}{l}\text { Lisozima, fagocitosis y actividad del } \\
\text { estallido respiratorio. Resistencia a } A \text {. } \\
\text { agalactie }\end{array}$ \\
\hline O. mossambicus & $\begin{array}{l}\text { Extracto de Cynodon } \\
\text { dactylon y Zingiber } \\
\text { officinale }\end{array}$ & $1 \%$ - 45 días & Fagocitosis y lisozima \\
\hline O. niloticus & $\begin{array}{l}\text { Extracto de Cratoxylum } \\
\text { formosum }\end{array}$ & 0.5 a $1.5 \%$ - 45 días & $\begin{array}{l}\text { Fagocitosis, estallido respiratorio y } \\
\text { lisozima }\end{array}$ \\
\hline O. niloticus & $\begin{array}{l}\text { Extracto de } \\
\text { Echinacea purpurea }\end{array}$ & Oral 0.25 ppt-168 días & $\begin{array}{l}\text { Incremento de leucocitos, lisozimas y } \\
\text { resistencia a Pseudomonas fluorescens }\end{array}$ \\
\hline O. niloticus & $\begin{array}{l}\text { Extracto de plantas china } \\
\text { (Astragalus membranaceus } \\
\text { y Lonicera japonica) }\end{array}$ & Oral $0.05-0.1 \%$ & $\begin{array}{l}\text { Estallido respiratorio, actividad } \\
\text { fagocítica, lisozima plamática, } \\
\text { proteína total e inmunoglobulina }\end{array}$ \\
\hline
\end{tabular}

Fuente: tomado de Quezada-Rodríguez (2012).

de formaldehído, comúnmente usados como antiparasitarios en salmónidos y peces de agua dulce. Recientemente, se incluyó el peróxido de hidrógeno $35 \%$ contra saprogleniasis en huevos, enfermedades branquiales bacterianas en salmónidos y peces de agua dulce de climas fríos, y cloramina $\mathrm{T}$ contra la flavobacteriosis. Los antibióticos aprobados son el florfenicol, la oxitetraciclina HCL y Dihidratada, así como la sulfadimetoxina con el ormetropim (FDA, 2016).

Otras drogas que representan un menor riesgo, tanto para el pez como para el consumidor y medio ambiente, son las que están agrupadas en la categoría de baja prioridad regulatoria, entre las cuales se encuentran la sal común, el ajo, la cebolla, el óxido de calcio, el ácido acético y el sulfato de magnesio. Las bases químicas de las drogas de este grupo son moléculas fácilmente degradables o neutralizables $\mathrm{y}$, en caso de acumulación en el medio natural, por su complejidad y efecto representan un menor riesgo sobre las especies blanco. En el cuadro 2 se relacionan algunos extractos de plantas naturales evaluados a nivel experimental o piloto contra parásitos y bacterias en tilapias. 
Cuadro 2. Extractos de plantas que se han evaluado contra parásitos y bacterias en tilapias.

\begin{tabular}{|c|c|c|c|}
\hline Nombre de la planta & ESPECIE DE TILAPIA & Patógeno & REFERENCIA \\
\hline Romero, albaca, guayaba, & O. aureus & Protozoarios & $\begin{array}{l}\text { Ocampo y } \\
\text { Auró, } 2000\end{array}$ \\
\hline Trueno & O. aureus & Costiasis & $\begin{array}{l}\text { Ocampo y } \\
\text { Auró, } 2000\end{array}$ \\
\hline Pino, ajo & O. aureus & $\begin{array}{l}\text { I. multifillis } \\
\text { Enfermedades bacterianas }\end{array}$ & $\begin{array}{l}\text { Ocampo y } \\
\text { Auró, } 2000\end{array}$ \\
\hline Paraíso, ajo & O. aureus & Cryptocaryon Neobenedenia & $\begin{array}{l}\text { Fernández, } \\
2003\end{array}$ \\
\hline Ajo, papaya, castaña & O. mossambicus & Nemátodos y acantocéfalos & $\begin{array}{l}\text { Ocampo y } \\
\text { Auró, 2000; } \\
\text { Auró, } 2004\end{array}$ \\
\hline Guayaba, pino y eucalipto & O. aureus & Enfermedades bacterianas & $\begin{array}{l}\text { Guerra et al., } \\
2001\end{array}$ \\
\hline
\end{tabular}

\section{Conclusiones}

Los avances alcanzados en el conocimiento de las enfermedades de la tilapia requieren de la aplicación de soluciones integrales orientadas al cumplimiento de las medidas de bioseguridad y buenas prácticas de producción para evitar la introducción y dispersión de patógenos. Las investigaciones sobre el efecto de la herbolaria medicinal en la prevención y control de las enfermedades deben ser una tarea prioritaria. La capacitación sistemática del personal técnico en la detección temprana de agentes patógenos garantiza la aplicación de medidas terapéuticas para su control.

\section{LITERATURA CITADA}

Agnew, W., A. C. Barnes. 2007. Streptococcus iniae: an aquatic pathogen of global veterinary significance and a challenging candidate for reliable vaccination. Veterinary Microbiology 122: 1-15.

Auró, O. 2004. Evaluación del efecto de las semillas de papaya (Carica papaya) sobre Cucullanus sp. en tilapia. REDVET 5, s/n.

Aly, S. M., F. M. Mohamed, G. John. 2008. Echinacea as immunostimulatory agent in Nile tilapia (Oreochromis niloticus) via earthen pond experiment. 8th International Symposium on Tilapia Aquaculture: 1033-1042.

Ardó, L., G. Yin, P. Xu, L. Varadi, G. Szigeti, Z. Jeney, G. Jeney. 2008. Chinese herbs (Astragalus membranaceus and Lonicera japonica) and boron enhance the non-specific immune response of Nile tilapia (Oreochromis niloticus) and resistance against Aeromonas hydrophila. Aquaculture 275: 26-33.

Arthur, J.R., M.G. Bondad-Rentaso, R.P. Subasinghe. 2012. Procedimientos para la cuarentena de animales acuáticos vivos: un manual. FAO Documento Técnico de Pesca y Acuicultura No. 502. Roma, Italia. 78 pp.

Cabello, F. C. 2006. Heavy use of prophylactic antibiotics in aquaculture: a growing problem for human and animal health and for the environment. Environmental Microbiology 8: 1137-1144.

Castro, R., I. Zarra, J. Lamas. 2004. Water-soluble seaweed extracts modulate the respiratory burst activity of turbot phagocytes. Aquaculture 229: 67-78. 
Comisión Nacional de Acuicultura y Pesca (Conapesca) 2015. Anuario Estadístico de Acuacultura y Pesca. Base de datos de producción 2014. México.

Costa, L. S. 2010. Biological activities of sulfated polysaccharides from tropical seaweeds. Biomedicine \& Pharmacotherapy 64: 21-28.

Cusack, R., D. K. Cone. 1985. A report of bacterial microcolonies on the surface of Gyrodactylus (Monogenea). Journal of Fish Diseases 8: 125-127.

del Rio-Zaragoza, O., E. Fajer-Ávila, P. Almazán-Rueda. 2011. Influence of $\beta$-glucan on innate immunity and resistance of Lutjanus guttatus to an experimental infection of dactylogyrid monogeneans. Parasite Immunology 33: 483-494.

El-Sayed, A. F. M. 2006. Tilapia culture. CABI Publishing Series: $277 \mathrm{pp}$.

Eyngor, M., R. Zamostiano, J. E. K. Tsofack, A. Berkowitz, H. Bercovier, S. Tinman, M. Lev, A. Hurvitz, M. Galeotti, E. Bacharach, A. Eldar. 2014. Identification of a novel RNA virus lethal to tilapia. Journal of Clinical Microbiology 52: 4137-4146.

Fernández, A. 2003. Evaluación de extractos de plantas medicinales con actividad antiparasitaria. CIVA. Disponible en: http://www.civa2003.org (consultado en marzo de 2017)

Food and Drug Administration (FDA). 2016. Approved Aquaculture Drugs. Disponible en: https://www.fda. gov/AnimalVeterinary/DevelopmentApprovalProcess/ Aquaculture/ucm132954.htm (consultado en marzo de 2017).

Fujiki, K., D. Shin, M. Nakao, T. Yano. 1997. Effects of ê-carrageenan on the non-specific defense system of carp Cyprinus carpio. Fisheries Science 63: 934-938.

García-Vásquez, A., H. Hansen, A. P. Shinn. 2007. A revised description of Gyrodactylus cichlidarum Paperna, 1968 (Gyrodactylidae) from the Nile tilapia, Oreochromis niloticus niloticus (Cichlidae), and its synonymy with G. niloticus Cone, Arthur et Bondad-Reantaso, 1995. Folia Parasitologica 54: 129-140.

García-Vásquez, A, H. Hansen, K. V. Christison, J. E. Bron, A. P. Shinn. 2011. Description of three new species of Gyrodactylus von Nordmann, 1832 (Monogenea) parasitizing Oreochromis niloticus niloticus (L.) and O. mossambicus (Peters) (Cichlidae). Acta Parasitologica 56: 20-33.

García O., A., O. Calvario M. 2007. Producción de peces. Pp. 283-309. In: Gardea B., A. A., G. A. González, I. Higuera-Ciapara \& F. Cuamea N. (Eds.). Buenas Prácticas en la Producción de Alimentos. Productos Pecuarios, Productos Agrícolas, Productos Acuícolas, Procesamiento de Alimentos. Trillas. México, D.F.

Gioacchini, G., P. Smith, O. Carnevali. 2008. Effects of ergosan on the expression of cytokine genes in the liver of juvenile rainbow trout (Oncorhynchus mykiss) exposed to enteric red mouth vaccine. Veterinary Immunology and Immunopathology 123: 215-222.

Guerra, O. M., I. D. Torres, P. L. Martínez. 2001. Validación del uso de plantas medicinales cultivadas en Cuba. Re- vista Cubana de Plantas Medicinales 2: 48-51.

Johnson, S. K. 2000. Protozoans as disease agents. pp. 695705. In: R. R. Stickney (Ed.) Encyclopedia of Aquaculture. John Wiley \& Sons. New York, USA.

Leiro, J. M., R. Castro, J. A. Arranz, J. Lamas. 2007. Immunomodulating activities of acidic sulphated polysaccharides from the seaweed Ulva rigida C. Agardh. International Immunopharmacology 7: 879-888.

Ocampo, C. L., O. A. Auró. 2000. Terapia de las enfermedades de los peces. SUA editorial, FMVZ-UNAM. México, DF. 42 pp.

Paperna, I. 1973. Lymphocystis in fish from East African lakes 1. Journal of Wild Diseases 9: 331-335.

Peddie, S., J. Zou, J. Secombes. 2002. Immunostimulation in the rainbow trout (Oncorhynchus mykiss) following intraperitoneal administration of Ergosan. Veterinary Immunology and Immunopatholology 86: 101-113.

Quezada-Rodríguez, P. R. 2012. Efecto dietario del extracto de Ulva clathrata sobre parámetros hematológicos, inmunológicos y crecimiento de tilapia (Oreochromis niloticus). Tesis de Maestría en Ciencias, Posgrado CIAD, A.C. 84 pp.

Rawson, M. V., W. A. Rogers. 1973. Seasonal abundance of Gyrodactylus macrochiri Hoffman and Putz, 1964 on bluegill and largemouth bass. Journal of Wild Diseases 9: 174-177.

Shlapobersky, M., M. S. Sinyakov, M. Katzenellenbogen, R. Sarid, J. Don, R. R. Avtalion. 2010. Viral encephalitis of tilapia larvae: primary characterization of novel herpes-like virus. Virology 399: 239-247.

Soto-Rodriguez, S. A., J. Cabanillas-Ramos, U. Alcaraz, B. Gómez-Gil, J. L. Romalde. 2013. Identification and virulence of Aeromonas dhakensis, Pseudomonas mosselii and Microbacterium paraoxydans isolated from Nile tilapia, Oreochromis niloticus, cultivated in Mexico. Journal of Applied Microbiology 115: 654-662.

Yin, G., G. Jeney, T. Raez, P. Xu, X. Jun, X. Jeney. 2005. Effect of two Chinese herbs (Astragalus radix and Scutellaria radix) on non specific immune response of tilapia, Oreochromis niloticus. Aquaculture 253: 39-47.

Xu, D. H., C. A. Shoemaker, P. H. Klesius. 2007. Evaluation of the link between gyrodactylosis and streptococcosis of Nile tilapia, Oreochromis niloticus (L.). Journal of Fish Diseases 30: 233-238.

Xu, D. H., C. A. Shoemaker, P. H. Klesius. 2009. Enhanced mortality in Nile tilapia Oreochromis niloticus following coinfections with ichthyophthiriasis and streptococcosis. Disease of Aquatic Organisms 85: 187-192.

Xu, D. H., C. A. Shoemaker, D. Zhang. 2015. Treatment of Trichodina sp reduced load of Flavobacterium columnare and improve survival of hybrid tilapia. Aquaculture Reports 2: 126-131.

Wu, C. C., C. H. Liu, Y. P. Chang, S. L. Hsieh. 2010. Effects of hot-water extract of Toona sinensis on immune response and resistance to Aeromonas hydrophila in Oreochromis mossambicus. Fish \& Shellfish Immunology 29: 258-263. 